\title{
Multiple memory traces after associative learning in the honey bee antennal lobe
}

\author{
Lisa Rath, C. Giovanni Galizia and Paul Szyszka \\ Department of Biology, University of Konstanz, D-78457 Konstanz, Germany \\ Keywords: associative learning, calcium-imaging, neural model, neural plasticity, olfaction
}

\begin{abstract}
We investigated the effect of associative learning on early sensory processing, by combining classical conditioning with in vivo calcium-imaging of secondary olfactory neurons, the projection neurons (PNs) in the honey bee antennal lobe (AL). We trained bees in a differential conditioning paradigm in which one odour $(\mathrm{A}+)$ was paired with a reward, while another odour (B-) was presented without a reward. Two to five hours after differential conditioning, the two odour-response patterns became more different in bees that learned to discriminate between $A$ and $B$, but not in bees that did not discriminate. This learning-related change in neural odour representations can be traced back to glomerulus-specific neural plasticity, which depended on the response profile of the glomerulus before training. (i) Glomeruli responding to $A$ but not to $B$ generally increased in response strength. (ii) Glomeruli responding to $B$ but not to $A$ did not change in response strength. (iii) Glomeruli responding to $A$ and $B$ decreased in response strength. (iv) Glomeruli not responding to $A$ or $B$ increased in response strength. The data are consistent with a neural network model of the $A L$, which we based on two plastic synapse types and two well-known learning rules: associative, reinforcer-dependent Hebbian plasticity at synapses between olfactory receptor neurons (ORNs) and PNs; and reinforcer-independent Hebbian plasticity at synapses between local interneurons and ORNs. The observed changes strengthen the idea that odour learning optimizes odour representations, and facilitates the detection and discrimination of learned odours.
\end{abstract}

\section{Introduction}

Learning the simple association between two stimuli gives rise to multiple memory traces in the brain. These memory traces are expressed as altered synaptic connections and neural excitability, evolve over time, and are distributed across brain areas (Menzel, 2001; Thompson, 2005; Blum et al., 2009). Here we ask how associative learning affects early sensory processing. We investigated the effect of associative odour-reward learning on odour processing in the honey bee primary olfactory area, the antennal lobe $(\mathrm{AL})$, which is the insect analogue of the vertebrate olfactory bulb (Hildebrand \& Shepherd, 1997). The insect olfactory system is a powerful model for studying mechanisms of learning and memory, because olfactory behaviour is highly plastic (Menzel \& Bitterman, 1983; Giurfa, 2007), and the neural representations of olfactory and appetitive or aversive reinforcing stimuli are well characterized (Davis, 2004; Schwaerzel \& Müller, 2006; Fiala, 2007). The ALs are subdivided into glomeruli with innate odour-response profiles (Galizia et al., 1999b). The output of a glomerulus is carried via projection neurons (PNs) to higher brain centres (Menzel et al., 2005). Within the AL a network of intra- and inter-glomerular inhibitory and excitatory local interneurons (LNs) is involved in odour processing (bee - Sachse \& Galizia, 2002; fly Shang et al., 2007; Silbering et al., 2008). Odour representations in

Correspondence: Dr P. Szyszka, as above.

E-mail: paul.szyszka@uni-konstanz.de
PNs are therefore the result of the interaction of many neurons in the AL. These interactions might sharpen or broaden odour tuning, alter concentration dependencies, change the synaptic gain, and generate or modify temporal patterns (bee-Sachse \& Galizia, 2002; Linster et al., 2005; Deisig et al., 2010; fly - Wilson et al., 2004; Shang et al., 2007; Silbering \& Galizia, 2007; Olsen \& Wilson, 2008; locust Perez-Orive et al., 2002). The AL is not only involved in sensory odour processing, but also in associative odour-reward learning (bee Hammer \& Menzel, 1998; fly - Thum et al., 2007). In this process octopaminergic neurons provide information about the reward (bee Hammer, 1993; fly - Schwaerzel et al., 2003). The coincidence of odour- and reward-induced activity leads to associative learning. Accordingly, associative odour learning affects odour representations in the AL (bee - Faber et al., 1999; Sandoz et al., 2003; Arenas et al., 2009; Fernandez et al., 2009; Denker et al., 2010; fly - Yu et al., 2004; moth - Daly et al., 2004) and in downstream areas as the mushroom bodies (bee - Faber \& Menzel, 2001; Szyszka et al., 2008). It is thought that learning-induced changes further optimize the representation of learned odours that might improve the detection and discrimination of the learned odour (Faber et al., 1999). However, the underlying mechanisms of neural plasticity in the AL are not well understood. We combined behavioural learning experiments with in vivo calcium-imaging, and investigated whether and how differential odour conditioning changes the neural representations in the uniglomerular PNs of the honey bee $\mathrm{AL}$. We found associative plasticity of odour representations $2-5 \mathrm{~h}$ after discriminative odour 
learning, which increased the distance between rewarded and unrewarded odours, and appeared as glomerulus-specific changes in PN response strength. These observations lead to a precise and testable prediction about which synaptic contacts are changed within the $\mathrm{AL}$ after appetitive conditioning.

\section{Materials and methods \\ Animals}

Experiments were performed with honeybee foragers (Apis mellifera).

\section{Staining and preparation}

The experiments took 2 days (Fig. 1A). The day before the experiments bees were caught from feeders near the hives, and the PNs of lateral and medial antenno-protocerebral tract (l- and m-APT) were stained with the calcium-sensitive dye Fura-2 dextran (Invitrogen, Molecular Probes, Eugene, OR, USA), as described in Szyszka et al. (2011; Fig. 1B). The next day (14-16 h after dye injection), bees were imaged, then trained and again imaged. Before imaging, the antennae were fixed temporarily with n-eicosan (Sigma Aldrich Chemie GmbH, Steinheim, Germany). The tracheae above the ALs were removed carefully. To reduce movements, the oesophagus and the surrounding muscles were lifted through a small opening in the clypeus, and fixed with two-component silicon (Kwik-Sil; World Precision Instruments, Sarasota, FL, USA), and the thorax and abdomen were gently pushed against the stage with a piece of foam $(1 \mathrm{~cm} \times 1 \mathrm{~cm} \times 3 \mathrm{~cm})$. The brain was covered with a thin layer of transparent Kwik-Sil (Okada et al., 2007). Within 10-30 min after the preparation bees were put under the microscope and heated to $24-28{ }^{\circ} \mathrm{C}$ with an infrared lamp. The $\mathrm{AL}$ that showed better staining and stronger spontaneous activity was chosen for measuring.

\section{Odour stimulation}

We used 2-octanol, 1-hexanol and 2-nonanol for odour stimulation (all from Sigma Aldrich). The pure odorants were diluted to $10^{-2}$ in mineral oil (Sigma Aldrich). Odorant dilutions were prepared freshly every 4 weeks. Two-hundred microlitres odour solution was loaded onto a cellulose stripe (Sugi, REF 31003; Kettenbach GmbH KG, Eschenburg, Germany) located in a 3-mL syringe (Norm-Ject; HenkeSass, Wolf GmbH, Tuttlingen, Germany). Syringes were prepared freshly every day. Odour stimuli were delivered as 4-s pulses with a custom-built computer-controlled six-channel olfactometer (Szyszka et al., 2011). The air stream through each channel was $300 \mathrm{~mL} / \mathrm{min}$ each controlled by a flowmeter (Analyt-MTC $\mathrm{GmbH}$, Müllheim, Germany). Odours were injected into a continuous carrier air stream $(1200 \mathrm{~mL} / \mathrm{min})$, which was directed to the bee via a glass tube $(0.7 \mathrm{~cm}$ in diameter). The six channels added up to $1800 \mathrm{~mL} / \mathrm{min}$. Thus, there was a total air stream of $3000 \mathrm{~mL} / \mathrm{min}$ directed at the bee positioned about $1 \mathrm{~cm}$ in front of the glass tube, corresponding to an air speed of $1.3 \mathrm{~m} / \mathrm{s}$. The binary mixture of 1-hexanol and 2-octanol was created by opening the valves of the two channels simultaneously. Continuous air suction behind the bee cleared residual odour.

\section{Imaging}

PNs in the AL were imaged through a water immersion objective (20×, NA 0.95; Olympus, Tokyo, Japan). The imaging system consisted of a fluorescence microscope (BX-50WI; Olympus), a light source (Polychrome IV; Till Photonics, Gräfelfing, Germany) and a CCD camera (Imago QE; Till Photonics); $8 \times 8$ pixels of the camera were binned on-chip resulting in a resolution of $172 \times 130$ pixels $(441 \times 333 \mu \mathrm{m})$. Each recording lasted $29 \mathrm{~s}$ and consisted of 232 double frames recorded with 340 - and 380 -nm excitation light at a rate of $8 \mathrm{~Hz}$. Excitation and emission light were separated with a 420-nm dichroic mirror and a 490-530-nm emission filter. Bees were stimulated with 2-octanol and 1-hexanol (odours used as $\mathrm{A}+$ and B- during training), their binary mixture, 2-nonanol and the solvent mineral oil as blank control. Odours were presented in a pseudorandomized sequence (all permutations were used) with an inter-trial interval of 2 min. Odour stimulation was controlled by the ACQUISITION software of the imaging system (Till Vision; Till Photonics).

\section{Differential conditioning}

Bees were differentially conditioned with 2-octanol and 1-hexanol as either rewarded odour $\mathrm{A}+$ or unrewarded odour B-. 2-Octanol and 1hexanol were used equally often as $\mathrm{A}+$. Bees received six training trials with $\mathrm{A}+$ and six training trials with $\mathrm{B}-$, presented alternately. In half of the bees training started with $\mathrm{A}+$ in the other half with $\mathrm{B}-$. The inter-trial interval between $\mathrm{A}+$ and $\mathrm{B}-$ was $10 \mathrm{~min}$. Bees were moved in front of the olfactometer $20 \mathrm{~s}$ before odour stimulation to habituate to the airflow. Odour pulses were $4 \mathrm{~s}$ long. For $\mathrm{A}+$ the odour was paired with sucrose stimulus (1.25 $\mathrm{M}$ in water), which was applied to the antenna and proboscis with a metal preparation needle. Sucrose stimulation started $3 \mathrm{~s}$ after odour onset and lasted for $3 \mathrm{~s}$. Thus, there was a 1-s overlap between odour and sucrose. A proboscis extension reflex (PER) was counted as response when the proboscis was extended horizontally. The training took $2 \mathrm{~h}$. Memory was tested $0.5 \mathrm{~h}$ after the last training trial. During the test, bees were exposed to A, B, the binary mixture $\mathrm{AB}$ and 2-nonanol $(\mathrm{N})$ as a new odour. The intertrial interval was again $10 \mathrm{~min}$, and the sequence of odour stimulation was pseudo-randomized (all permutations were used).

\section{Experimental protocol}

PNs were stained on the first day (Fig. 1A). On the second day, bees were differentially conditioned, and their learning and memory performances were measured behaviourally. One-nine hours after PN imaging bees were conditioned. Two-five hours after the last training trial PN responses were imaged again. Ninety bees were stained and conditioned. In 25 of these bees, PN responses could be measured until the end of the imaging experiment. Bees were classified as discriminators (more responses to A than to B in the 2nd6th training and test trials) or non-discriminators (equal or fewer responses to A than to B; Roussel et al., 2010). There were 12 discriminators (six received 1-hexanol, six received 2-octanol as $\mathrm{A}+$ ) and 13 non-discriminators (nine received 1-hexanol, four received 2octanol as $\mathrm{A}+$ ).

\section{Data analysis}

Imaging data were analysed with custom-written programs in IDL (RSI, Boulder, CO, USA). First, measurements were movement corrected by aligning frames within and between measurements. Then, glomeruli were segmented with the help of an unsharp masked image of the raw fluorescence and a correlation image where the correlation of the signal traces between neighbouring pixels was calculated (Fig. 1D). Glomeruli were identified by comparing glomerulus position and size with the morphological atlas of the honeybee (Galizia et al., 1999a) 

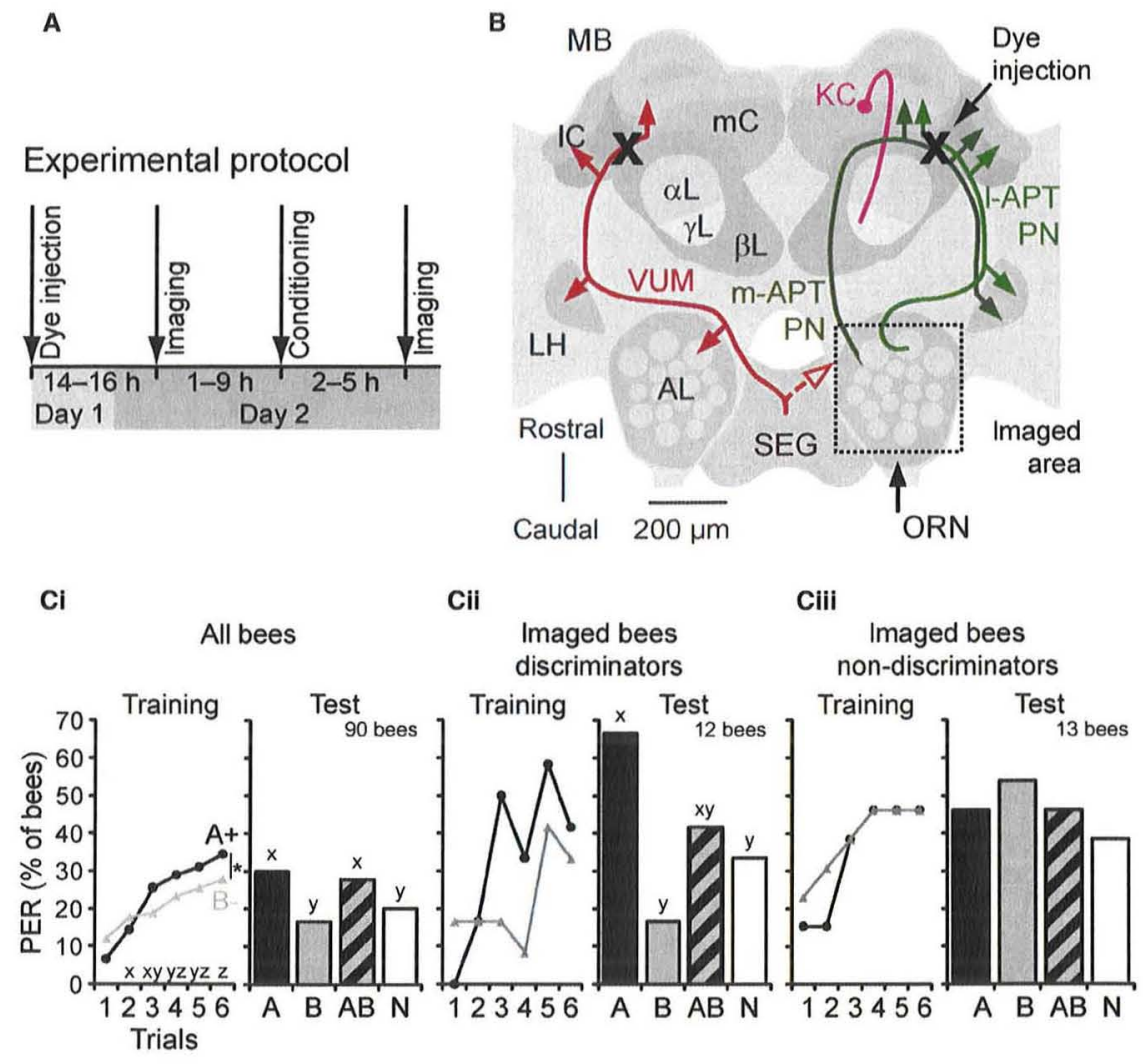

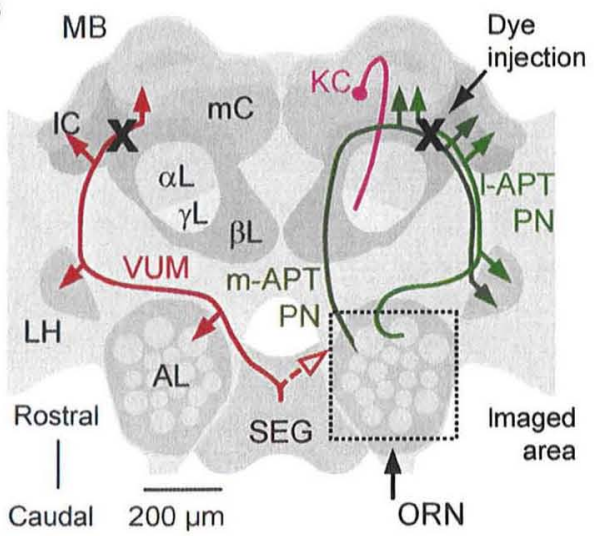

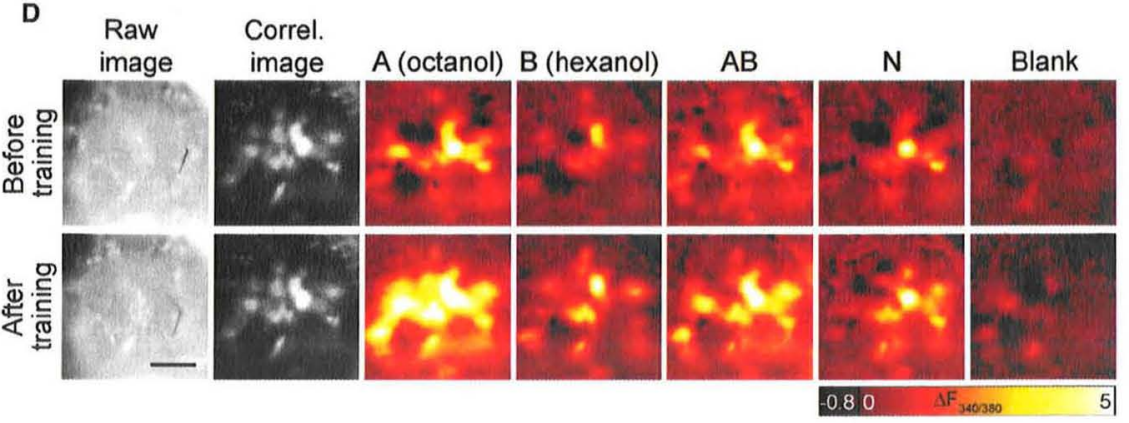

FIG. 1. Bees learn after disrupting PN axons and imaging. (A) Scheme of the experimental protocol. (B) Frontal view of the honey bee central olfactory system. Different neurons are shown in the two hemispheres. Olfactory receptor neurons (ORN, black arrow) terminate in the antennal lobe (AL) glomeruli. Uniglomerular projection neurons (PN, green) transmit odour information from the AL to the mushroom body (MB) and to the lateral horn (LH). PN axons project along the lateral or median antenno-protocerebral tract (1-APT, $\mathrm{m}$-APT) to the lateral and medial calyces (IC, mC). The MB intrinsic Kenyon cells (KC, magenta) send their axons into the MB lobes $(\alpha \mathrm{L}, \gamma \mathrm{L}, \beta \mathrm{L})$. In this study mostly l-APT PN dendrites that ramify in glomeruli in the frontal AL were imaged. The dotted square indicates the imaged area. The crosses indicate the sites of dye injection. Left - the bilaterally symmetrical VUM $_{m x \mid}$ neuron (VUM, red) mediates the reinforcing function of the reward and connects the sub-oesophageal ganglion (SEG) with the AL, LH and MB calyces. (C) Odour-evoked PER during training and memory retrieval test 30 min after. (Ci) Learning and memory performance of bees treated equally to the imaging bees. Training - PER responses increased stronger to A+ than B- (twoway RM ANOVA, $F_{\text {trial, } 4,356}=5.1, P<0.001 ; F_{\text {odour. } 1,356}=5.8, P=0.02 ; N=90$ bees; trials $\left.2-6\right)$. Test - the response frequencies to the CS+ and the mix were higher than to the CS - and N (one-way RM ANOVA, $F_{\text {odour. } 3,267}=5.4, P<0.001$, Holm-Sidak post hoc tests). (Cii, Ciii) Learning and memory performance of bees that were imaged before and after conditioning (this is a subset of the bees in Ci). These bees were used to investigate learning-induced changes in PN responses. Bees were classified as discriminators (Cii; more A than B responses during the 2nd-6th training and test trials) or non-discriminators (Ciii; equal or less A than B responses). The test confirms the separation into learners and non-learners - in discriminators the response frequencies to the CS+ were higher than to the CS- (one-

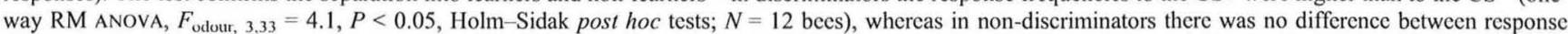
frequencies (one-way RM ANOVA, $F_{\text {odour } 3.36}=1.4, P=0.27 ; N=13$ bees). Different letters mark a significant difference $(P<0.05$ ). (D) Raw fluorescence and correlation images of the stained $\mathrm{AL}$ (left hemisphere) of a discriminator and colour-coded images of responses to $\mathrm{A}, \mathrm{B}$, the mixture $\mathrm{AB}$, nonanol (N) and the blank control before and after training (mean of $4 \mathrm{~s}$ during stimulus). Scale bar $-100 \mu \mathrm{m}$. For interpretation of color references in figure legend, please refer to the Web version of this article.

and the odour responses with the physiological atlas (Galizia et al., 1999b; Sachse et al., 1999). Signals were calculated as $F_{340} / F_{380}$. The baseline was shifted to 0 by subtracting the average signal before stimulation (frame 4-69 for odour stimulation). Activity patterns are shown as colour-coded images of single measurements, which were filtered with a spatial low-pass filter of $5 \times 5$ pixels for better visualization (Fig. 1D). No filtering was used for quantitative analysis. Glomerulus responses were calculated from a $5 \times 5$ pixels square in 
the glomerulus centre. Response strength was quantified as the mean signal during $4 \mathrm{~s}$ after stimulus onset (frames 72-103; colour-coded activity images and bar graphs in Figs 1-3). To calculate the global response strength across the entire $\mathrm{AL}$, for each bee, the response strength of each glomerulus was calculated and averaged over all glomeruli (Fig. 2A). The distance between odour response patterns was quantified as follows: for each bee, the response strength of each glomerulus was calculated and the odour response pattern was represented as a vector of these values. The distance between two odour responses was quantified by calculating the Euclidean distance between the glomerular odour-response vectors (Fig. 2B). In Fig. 3 each glomerulus was grouped according to its responses to the odours $\mathrm{A}$ and $\mathrm{B}$ before training. For each glomerulus, odour-evoked signals (mean signal during $4 \mathrm{~s}$ after odour onset) were classified as responses when the signal was three times larger than the standard deviation of the background activity of the glomerulus. The standard deviation was calculated over 66 frames before stimulation and averaged over five measurements (A, B, AB, N and blank). Odour-evoked signals below three times standard deviation were classified as weak or no responses. Behavioural data were analysed with an ANOVA. Although a parametric ANOVA test is usually not applicable for dichotomous data, an empirical study has shown that ANOVA is appropriate under our conditions (Lunney, 1970). However, for the data shown in Fig. 2Cii and iii, the power of the ANOVA is low because of the small sample size. Statistical tests were performed with R (http://www.r-project.org), and Sigma Stat (SPSS, Chicago, IL, USA).

\section{Results}

We combined differential conditioning with in vivo calcium-imaging of PNs to identify learning-related changes in PN responses. We stained uniglomerular PNs that send their axons through either the m-APT or l-APT (Abel et al., 2001; Kirschner et al., 2006; Galizia \&

FIG. 2. PN response patterns for the rewarded (A) and unrewarded odour (B) become more distinctive after differential conditioning. (A) Time-course of global PN responses (average over all glomeruli) in discriminators (Ai) and non-discriminators (Aii; mean $\pm \mathrm{SEM}$ ). The response to the mixture (32 frames during odour stimulation, before training) was higher than to all other odours (two-way RM ANOVA with factors 'odour' and 'frame'; $F_{\text {odour, } 4,2976}=28.8$,

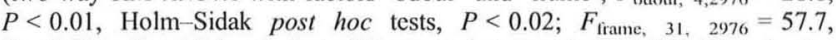
$P<0.001 ; N=25$ bees), but differences between before (black) and after the training (red) were not significant [two-way RM ANOVA with factors 'training' (before vs. after training) and 'frame'; discriminators $-F_{\text {training, }}$ $1.341=0-2.9, P=0.11-0.84 ; N=12$ bees; non-discriminators $-F_{\text {training. }}$ $1.372=0-0.52, P=0.48-0.99 ; N=13$ bees]. Stimulus bars indicate the 4-S odour pulse. (B) Time-resolved Euclidean distance between glomerular PN response patterns before (black) and after conditioning (green; mean \pm SEM). (Bi) In discriminators the distance during the odour stimulation (32 frames) increased between the two odour-response patterns after training [two-way RM ANOVA with factors 'training' (before vs. after training) and 'frame'; $F_{\text {training, }}$ ${ }_{1341}=11.2, P<0.01 ; F_{\text {taining }}$ iname, 31.341 $\left.=1.6, P<0.05\right]$. The distance between the mixture $\mathrm{AB}$ and either $\mathrm{A}$ or $\mathrm{B}$ did not change after training (twoway RM ANOVA; $\mathrm{A}$ vs. $\mathrm{AB}-F_{\text {training, } 1,341}=2.9, P=0.12 ; \mathrm{B}$ vs. $\mathrm{AB}-F_{\text {training, }}$ $1,341=0.9, P=0.36$ ). (Bii) In non-discriminators there was no change in pattern distances after training (two-way RM ANOVA; A vs. B $-F_{\text {training. }}$ $1,341=1.8, P=0.2 ; \mathrm{A}$ vs. $\mathrm{AB}-F_{\text {training, } 1,341}=2.7, P=0.12 ; \mathrm{B}$ vs. $\mathrm{AB}-$ $\left.F_{\text {training, }, 341}=0.5, \quad P=0.5\right)$. Asterisks mark significant difference $(* * P<0.01)$. (C) PN response strength (mean of $4 \mathrm{~s}$ during stimulus $\pm \mathrm{SEM}$ ) in identified glomeruli for the rewarded odour A (top) and the unrewarded odour B (bottom) before and after training. The rewarded odour A was either hexanol (left) or octanol (right). There were no consistent changes in response strength after training in identified glomeruli (paired $t$-test; $P>0.23$ if not otherwise stated). The glomerulus identity is indicated at the bottom. Numbers in brackets indicate number of bees. Only glomeruli are shown that were recorded in at least three bees. For interpretation of color references in figure legend, please refer to the Web version of this article.
Rössler, 2009; Fig. 1B). We measured the frontal part of the AL where 1-APT innervated glomeruli are visible. Thus, even though we stained both tracts, we imaged predominantly l-APT glomeruli. We chose differential classical conditioning in order to identify excitatory and

Ai Discriminators Ail Non-discriminators

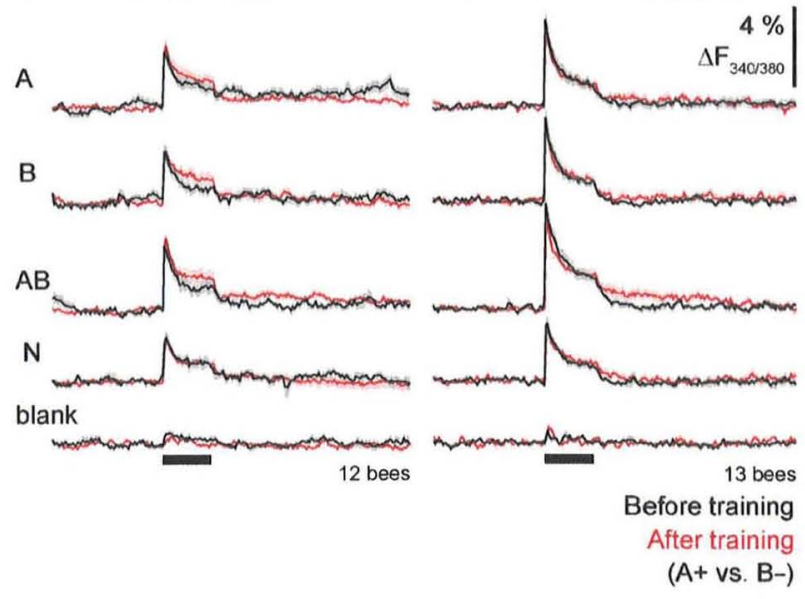

$\mathrm{Bi}$

Discriminators

Bii Non-discriminators

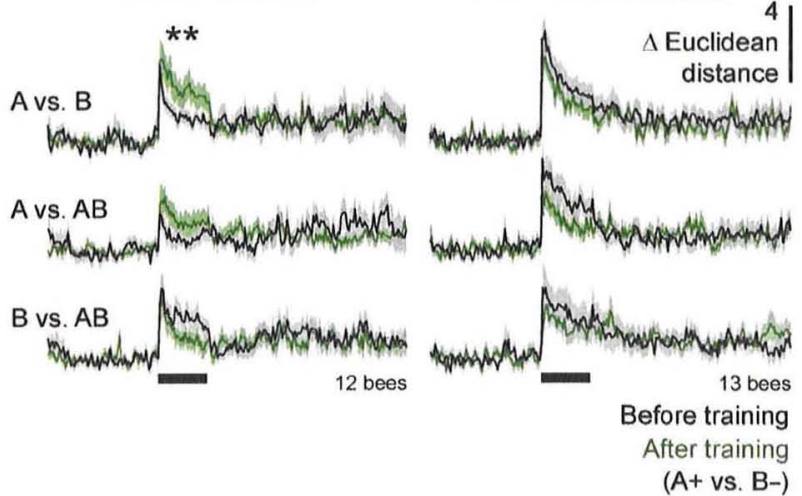

C

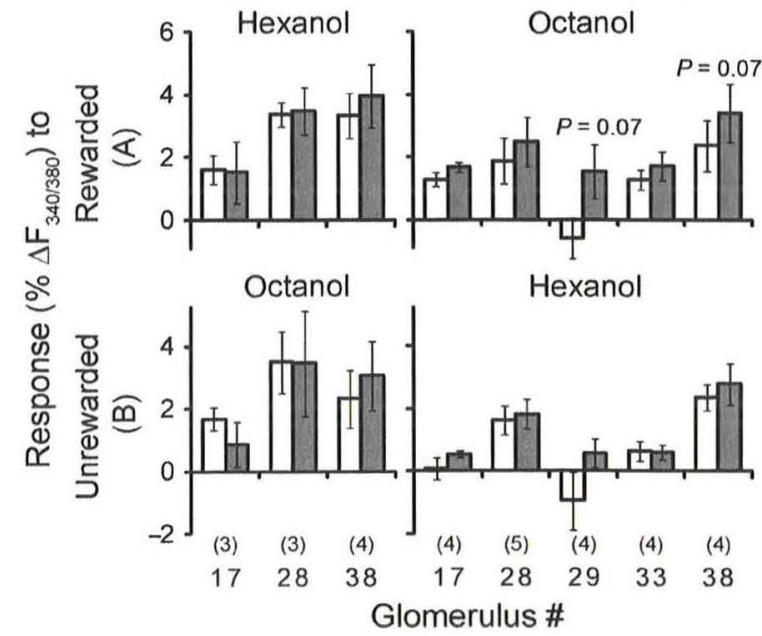

$\square$ Before training

After training

(A+ vs. B-) 


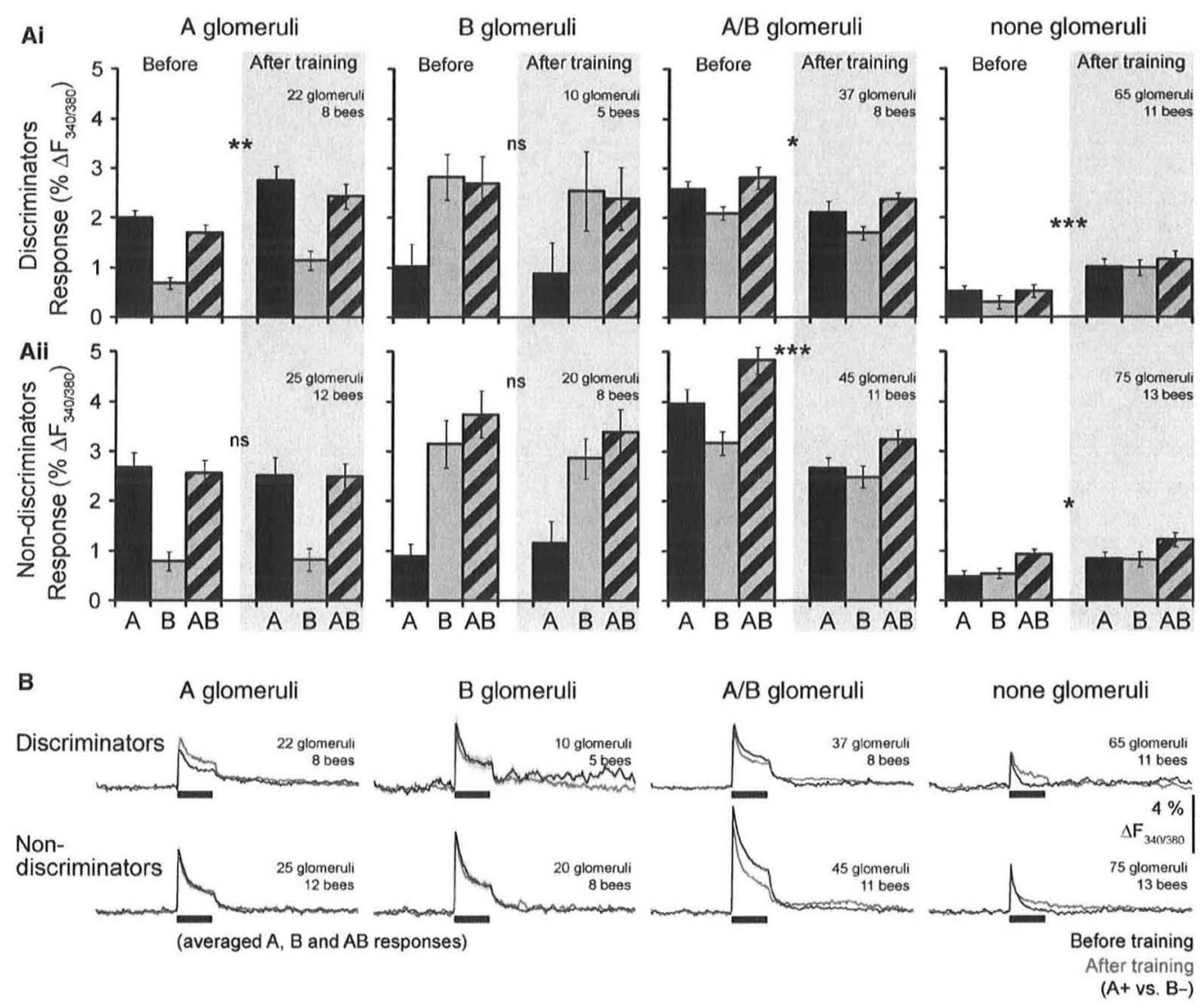

FIG. 3. Associative plasticity in PN responses depends on the glomerulus response profiles. (A) PN response strength (mean of $4 \mathrm{~s}$ during stimulus \pm SEM) before and after training in discriminators (Ai) and non-discriminators (Aii). Each glomerulus was divided into one of four groups according to its response profile before conditioning. (Ai) In discriminators 'A glomeruli' increased in response strength to A, B and the mixture AB [two-way RM ANOVA with factors odour and training (before vs. after training), $F_{\text {trainine } 1.42}=11.4, P<0.01 ; F_{\text {training }} \times$ odours, $2.42=1.7, P=0.2 ; N=22$ glomeruli]. 'B glomeruli' did not change in response strength

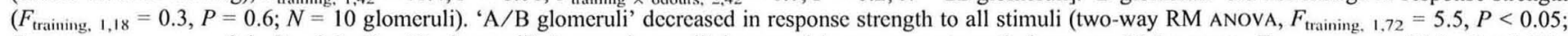
$F_{\text {training }} \times$ odours. $2.72=0.1, P=0.9 ; N=37$ glomeruli). 'none glomeruli' increased in response strength (two-way RM ANOVA, $F_{\text {training, } 1,128}=21.9, P<0.001$; $F_{\text {training }} \times$ odours, $2,128=1.6, P=0.2 ; N=65$ glomeruli). (Aii) In non-discriminators neither 'A glomeruli' nor 'B glomeruli' changed in response strength [two-way RM ANOVA with factors odour and training (before vs. after training), 'A glomeruli' $-F_{\text {training. } 1.48}=0.2, P=0.7 ; N=25$ glomeruli; 'B glomeruli' $-F_{\text {training, }}$ $1,38=0.2, P=0.7 ; N=20$ glomeruli]. 'A/B glomeruli' decreased in response strength $\left(F_{\text {training, }}\right.$ 1,88 $=37.3, P<0.001 ; F_{\text {training }} \times$ odours, $2,88=7.6, P<0.001 ;$

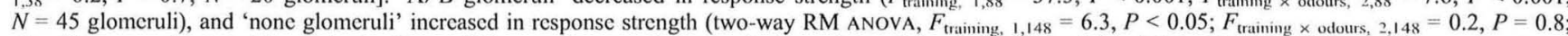
$N=75$ glomeruli). Asterisks mark significant differences between before and after training $(* P<0.05 ; * * P<0.01 ; * * * P<0.001)$. (B) Mean PN response traces before (black) and after training (red) in discriminators (top) and non-discriminators (bottom). Because response changes were not odour-stimulus dependent, we averaged the traces for $\mathrm{A}, \mathrm{B}$ and $\mathrm{AB}$ in order to increase the signal-to-noise ratio. Shaded areas indicate \pm SEM. Stimulus bars indicate the 4-s odour pulse. For interpretation of color references in figure legend, please refer to the Web version of this article.

inhibitory learning effects. In this paradigm one odour is rewarded $(\mathrm{A}+)$ and associated with the reward (excitatory learning), whereas another odour is presented alone (B-) and associated with the absence of reward (inhibitory learning). During the memory retrieval test and during the two PN imaging sessions before and after training (Fig. 1A) we measured responses to $\mathrm{A}$ and $\mathrm{B}$ and additionally to their binary mixture and to nonanol $(\mathrm{N})$, which was not presented during training in order to establish whether odour learning affects the representation of these odours. In this text, we refer to A and B as the rewarded and non-rewarded odours, respectively. In the context of acquisition, we refer to $\mathrm{A}^{+}$(paired with reward) and $\mathrm{B}-$ (not paired with reward).

\section{Associative odour learning is possible after disrupting the PN tracts by dye injection}

Because we used an invasive staining procedure (Fig. 1B), which might interfere with the bees' ability to learn or recognize odours, we first ascertained whether bees were able to learn after PN staining and imaging (Fig. 1Ci). During training, the proportion of bees showing the conditioned response (PER) increased. However, PER frequency only differed slightly between $\mathrm{A}+$ and $\mathrm{B}-(P=0.02)$. In the memory retrieval test $30 \mathrm{~min}$ after training, bees had higher response rates to $\mathrm{A}$ and $\mathrm{AB}$ than to $\mathrm{B}$ and nonanol $(P<0.001)$. Thus, bees were still able to learn after disrupting their PN tracts by dye injection and after the imaging procedure. However, as compared with intact bees (data not shown), differentiation between $\mathrm{A}$ and $\mathrm{B}$ was poor.

Differential odour conditioning increases the distance between the rewarded and unrewarded odour

Having established that bees with stained PNs can still learn to associate an odour with a sucrose reward, we investigated whether differential conditioning alters odour representations in the AL. We therefore recorded dendritic PN responses in the AL glomeruli before 
and after differential conditioning. In order to separate associative from non-associative effects of the training procedure, we divided bees into discriminators (Fig. 1Cii) and non-discriminators (Fig. 1Ciii). Separating bees into discriminators and non-discriminators considers the fact that associative learning performance differs across individual bees and does not reflect the mean population performance (Pamir et al., 2010). Accordingly, such separation proved useful in previous studies that investigated the effect of odour learning on neural odour representations in honey bees (Okada et al., 2007; Roussel et al., 2010).

Odour stimuli evoked glomerular patterns of increased or decreased calcium signals (Fig. 1D). The global response showed a phasic-tonic time-course, and was strongest for the mixture $\mathrm{AB}$ (Fig. 2A). The stronger response to the mixture is most likely due to the fact that the mixture was presented as the sum of octanol and hexanol, and thus activates more glomeruli. The global response did not change significantly between before and after training in both discriminators (Fig. 2Ai) and non-discriminators (Fig. 2Aii). We next asked whether differential conditioning modulates glomerular response patterns, and analysed the distance between the response patterns of $\mathrm{A}, \mathrm{B}$ and $\mathrm{AB}$ before and after the training. We quantified the distance between response patterns as Euclidean distance (Fig. 2B). In discriminators the distance between $\mathrm{A}$ and $\mathrm{B}$ increased after training, while the distance change between $\mathrm{AB}$ and either $\mathrm{A}$ or $\mathrm{B}$ was not significant (Fig. 2Bi). No significant change in inter-odour distances was detectable in non-discriminators (Fig. 2Bii). Thus, only in discriminators there was a differential change in PN response patterns, which made the $\mathrm{A}$ and $\mathrm{B}$ representation more distinctive after training.

\section{Associative plasticity in $P N$ responses depends on glomerulus response profiles}

The differential changes in PN response patterns imply unequal changes across glomeruli. Are these changes predictable across animals based on the identity of glomeruli? In 11 of the 12 discriminators we could identify glomeruli according to the morphological and physiological AL atlas (Galizia et al., 1999a; Sachse et al., 1999). We did not find consistent training-induced changes in response strength (Fig. 2C), confirming previously published data (Peele et al., 2006; Fernandez et al., 2009). However, in addition to stereotypical glomerular responses, there is also variability across animals. We therefore analysed glomerular response changes on the basis of their individual response to the odours used. For each individual bee, we classified glomeruli into four types according to their responses to $\mathrm{A}$ and $\mathrm{B}$ before training, and compared the responses with the two odours and their mixture before and after differential conditioning (Fig. 3). 'A glomeruli' - glomeruli with a response to $\mathrm{A}$ and no or weak response to $\mathrm{B}$; 'B glomeruli' glomeruli with a response to $\mathrm{B}$ and no or weak response to $\mathrm{A}$; ' $\mathrm{A} / \mathrm{B}$ glomeruli' - glomeruli with responses to both $\mathrm{A}$ and $\mathrm{B}$; 'none glomeruli' - glomeruli with no or weak response to both A and B. In discriminators, these glomerulus types showed differential changes in their response strength after the training (Fig. 3Ai). 'A glomeruli' increased in response strength equally to all test odours (ANOVA revealed no interaction between the factors 'training' and 'odours'). This increased response persisted for the entire stimulus length (see time-trace in Fig. 3B). 'B glomeruli' did not change in response strength. 'A/B glomeruli' decreased in response strength to all odours. This decrease persisted during the entire response time. 'None glomeruli' increased in response strength to all odours. This increase was strongest towards the end of the odour response, suggesting that it is caused by a different mechanism than the previous effects, thus implying at least two synaptic processes in the network. In nondiscriminators, neither 'A glomeruli' nor ' $\mathrm{B}$ glomeruli' changed in response strength; however, 'A/B glomeruli' and 'none glomeruli' showed the same training-induced changes as in discriminators and decreased and increased in response strength, respectively (Fig. 3Aii). These data show that there were differential changes in response strength across glomeruli after differential conditioning, and that these changes depended on the glomerulus response profiles and on whether the bee learned to discriminate between the rewarded and unrewarded odour.

\section{Discussion}

Odour representations in the primary olfactory area of insects and mammals are subject to experience-dependent plasticity. Various studies reported learning-induced changes in odour representations in the insect AL (bee - Faber et al., 1999; Sandoz et al., 2003; Arenas et al., 2009; Fernandez et al., 2009; Denker et al., 2010; fly - Yu et al., 2004; moth - Daly et al., 2004) and the mammalian olfactory bulb (Kay \& Laurent, 1999; Doucette \& Restrepo, 2008). However, a mechanistic understanding of associative plasticity of odour representations is still missing. To shed light on the rules under which associative learning changes the functional AL circuitry, we combined behavioural learning experiments with in vivo calcium-imaging of selectively stained PNs in honey bees. We found associative plasticity of odour representations after differential conditioning. In bees that learned to discriminate between the rewarded odour $\mathrm{A}$ and unrewarded odour B, plasticity differentially affected A and B responses, and was apparent as glomerulus-specific changes in PN response strength. Moreover, the learning-induced changes in a glomerulus could be predicted from its response profile before training.

\section{Methodological considerations}

We stained PNs by injecting a calcium indicator into the PN axon tracts between the mushroom body calyces of both hemispheres (Fig. 1B). This staining procedure disrupts PN axons and disturbs their connection to the mushroom bodies. Because the mushroom bodies play an important role in learning and memory (Menzel, 2001), our staining procedure might interfere with odour-reward learning. Indeed, the reduced odour discrimination of the imaged bees (Fig. 1) compared with intact animals is in line with mushroom bodies' suggested role in discriminative odour learning (Heisenberg, 2003). Moreover, PN output to the mushroom bodies appears to be required for memory retrieval, as cutting PN axons upstream to the mushroom body calyces blocks memory retrieval (Peele et al., 2006). Nevertheless, our bees were still able to learn and form an associative memory during differential conditioning (Fig. 1C). We assume that this ability derives from the fact that our staining procedure disrupted only a part of the connections between the ALs and mushroom bodies. It did not damage connections between the m-APT PNs and medial calyx, and between the 1-APT PNs and lateral calyx.

We used a training protocol that induces multiple, dynamic memory phases (Hammer \& Menzel, 1995). We recorded PN responses during a period of $2-5 \mathrm{~h}$ after conditioning, corresponding to mid-term memory, which requires the formation of a constitutively active protein kinase C within the AL (Grünbaum \& Müller, 1998). Before the second imaging session we performed one memory retrieval test. This presentation of the learned odour without a reward may induce additional learning processes like extinction and reconsolidation of the 
A

\begin{tabular}{|c|c|c|}
\hline Glomerulus type & $\begin{array}{l}\text { Response change } \\
\text { after conditioninng } \\
\text { (A+ vs. B-) }\end{array}$ & (1) ORN-to-PN syr \\
\hline A glomerulus & $\uparrow$ & $\begin{array}{l}\text { ORN } \\
\text { (excit.) LTP }\end{array}$ \\
\hline B glomerulus & $=$ & \\
\hline AB glomerulus & $\downarrow$ & $\begin{array}{l}\text { Active } \\
\text { Non-active }\end{array}$ \\
\hline None glomerulus & $\uparrow$ & $\begin{array}{l}\mathbb{T} \begin{array}{l}\text { Synaptic } \\
\text { strengthening }\end{array} \\
\text { Synaptic } \\
\Downarrow \text { weakening }\end{array}$ \\
\hline
\end{tabular}

Synaptic learning rules

(2) LN-to-ORN synapse
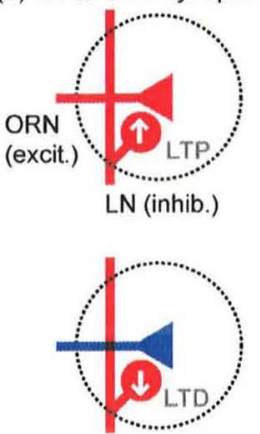

C

Learning induced changes in intra-glomerular synaptic connections

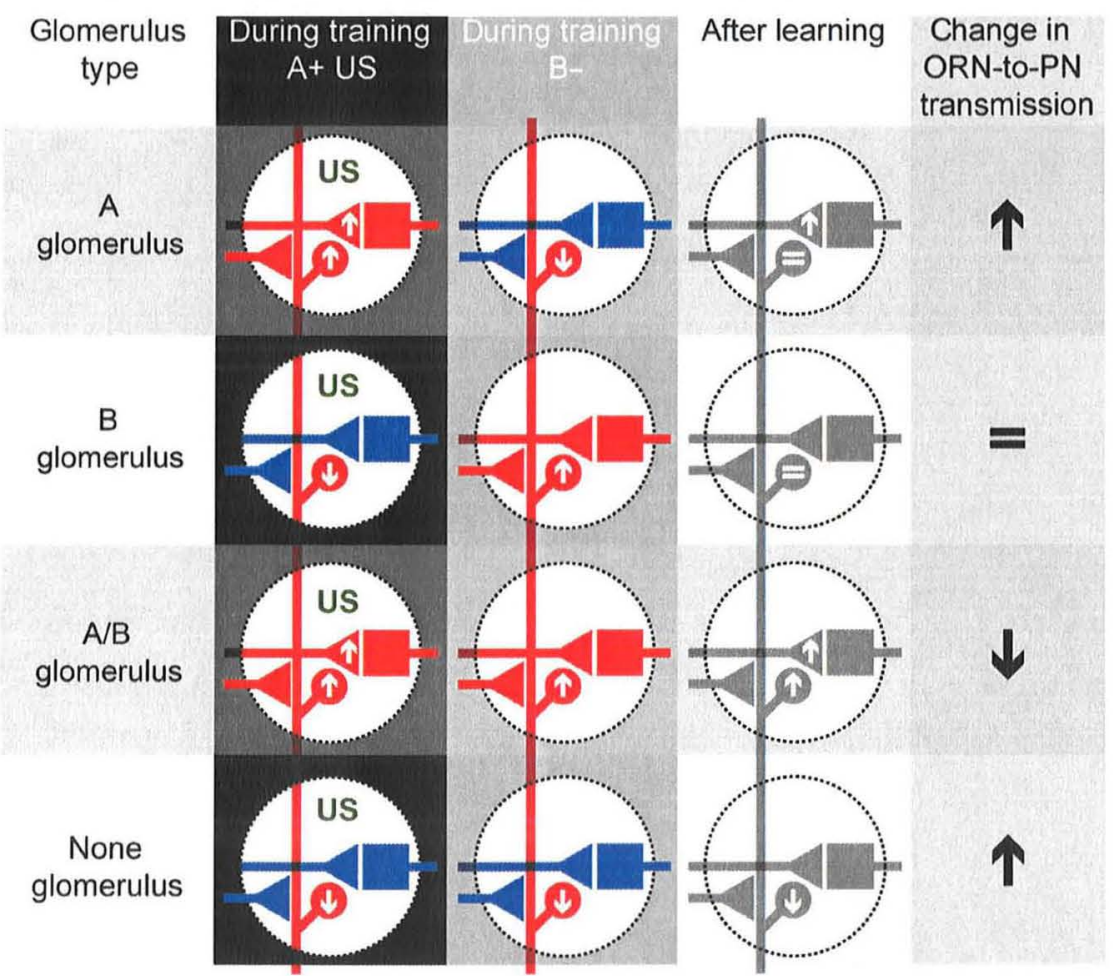

FiG. 4. Model of associative plasticity in the AL after differential conditioning. (A) Summary of changes in glomerulus response strength after differential conditioning (data from Fig. 3Ai). Equal signs indicate no change in response strength, arrows indicate significant changes and their direction. (B) Synaptic learning rules are as follows. (i) LTP at the excitatory ORN-to-PN synapse under the control of the unconditioned stimulus (US, reward) as a positive reinforcer. Coincident activity (red) at the ORN-to-PN synapse will strengthen synapses (arrow up) only if the US is present. (ii) Reinforcer-independent Hebbian LTP/LTD at the inhibitory LN-to-ORN synapsc. Coincident pre- and postsynaptic activity (red) leads to LTP. No activity (blue) in the postsynaptic ORN and activity (red) in the presynaptic LN leads to LTD. (C) Model of learning-induced plasticity in the AL. First row - 'A glomerulus'. The ORN-to-PN synapses are strengthened during the pairing of A+ with the US during training (rule 1). The LN-to-ORN synapses are strengthened during A+ presentation and weakened during B-presentation (rule 2), leading to no net change. Together, this results in an increased ORN-to-PN transmission (right column). Second row - 'B glomerulus'. The ORN-to-PN synapses do not change because there is no coincidence of ORN activity and the US. The LN-to-ORN synapses are weakened during A+ presentation and strengthened during Bpresentation (rule 2), summing up to no change in ORN-to-PN transmission. Third row - 'A/B glomerulus'. The ORN-to-PN synapses are strengthened during A+ presentation (rule 1). The LN-to-ORN synapses are strengthened during $\mathrm{A}+$ and $\mathrm{B}$ - presentation, and the increased inhibitory presynaptic output onto ORNs outweighs the increased strength of the ORN-to-PN synapse (rule 2). This sums up to a decreased ORN-to-PN transmission. Last row - 'none glomerulus'. The ORN-to-PN synapses do not change because there is no coincidence of ORN activity and the US (rule 1). The LN-to-ORN synapses are weakened because there is no postsynaptic activity (rule 2). This will lead to an increased ORN-to-PN transmission. For interpretation of color references in figure legend, please refer to the Web version of this article.

acquisition memory. Therefore, the observed training effects in PN responses might stem from combined effects of memory acquisition, extinction and reconsolidation. In the present study we cannot unravel the potential contribution of these learning processes to the observed effects. However, we assume that they are dominated by the associative memory acquisition because, in honey bees, both extinction and reconsolidation are absent or only weakly induced after a single retrieval trial (Stollhoff et al., 2005, 2008). 


\section{Differential odour conditioning improves separability of neural odour representations}

In discriminator bees, the neural representations of the rewarded odour $\mathrm{A}$ and the unrewarded odour $\mathrm{B}$ became more distinct after training (Fig. 2B). This finding is consistent with previous calcium-imaging studies in honey bees that found increased distances between PN responses to the rewarded and unrewarded odour after appetitive differential conditioning (Faber et al., 1999; Fernandez et al., 2009). Different to the findings of Faber et al., we did not observe increased global responses towards the rewarded odour. Because Faber and colleagues performed neuron-type unspecific bulk-staining, the learning-induced increase in response strength might reflect activity in other neurons than PNs. Our data contradict a previous study by Peele et al. (2006) who did not find changes in PN responses after differential conditioning. There are some differences between ours and Peele's study, which could explain this discrepancy, as follows. (i) Peele and colleagues measured PN responses 5-13 min after training, whereas we measured $2-5 \mathrm{~h}$ after training. Thus, the learning effects that we found might require consolidation time of more than 13 min. (ii) Peele's staining procedure disrupted the axons of l-APT PNs and the reward-mediating $\mathrm{VUM}_{\mathrm{mx} 1}$ neuron axons in the lateral protocerebrum, and thus cut their connection to the mushroom body (Hammer, 1993). This lesion might prevent memory acquisition. (iii) Peele et al. conducted their experiments at room temperature, while we heated the bees to $24-28{ }^{\circ} \mathrm{C}$. The higher temperature might strengthen the effects of learning-induced changes in synaptic weights.

\section{Glomerular response changes can be predicted from their response profile before training}

We were not able to relate the observed changes to identified glomeruli (Fig. 2C). This may indicate that associative odour learning affects different glomeruli in different animals. Indeed, given the finding that the neural odour representations are plastic, it is likely that different previous experience of the individual experimental animals may already have shaped their olfactory networks. Likewise, previous studies in honey bees failed to attribute PN response changes to the identity of glomeruli (Peele et al., 2006; Fernandez et al., 2009). However, we found associative plasticity in PNs that depended on the response profile of the innervated glomerulus before training (summarized in Fig. 4A). Where in the AL network do the glomerulus-type dependent changes occur? Glomeruli are interconnected by a dense network of LNs in bees (Galizia, 2008), some of which are global and some selective in their connectivity. Increasingly more LN subpopulations are being described (Kreissl et al., 2010). Synaptic contacts abound between olfactory receptor neurons (ORNs), PNs and LNs, and the octopaminergic $\mathrm{VUM}_{\mathrm{mx}}$ neuron that signals the reward in appetitive odour learning (Hammer, 1993). Given the large number of possibilities, our experiments are not suitable to identify the plastic synapse within the network. We can, however, propose a minimal network that is sufficient to explain the observed changes, as a working hypothesis for future research. We started by observing a differential effect early in the response as compared with late in the response (compare 'A glomeruli' with 'none glomeruli' in Fig. 3B). Furthermore, we found that some changes were common to discriminators and non-discriminators, while others were not. This prompted us to postulate two synaptic processes, one affecting the ORN-to-PN synapses directly and related to associative memory traces, the other affecting inhibitory LN-to-ORN synapses related to non-associative memory. These two mechanisms follow two distinct learning rules (Fig. 4B). (i) Long-term potentiation (LTP) at excitatory ORN-to-PN synapses under control of the $\mathrm{VUM}_{\mathrm{mx}}$. ORN-to-PN synapses undergo
LTP if the ORNs and PNs are active at the same time (Hebb, 1949) and the $\mathrm{VUM}_{\mathrm{mx}}$ signals the presence of a reward (Fig. $4 \mathrm{C}$, 'A glomerulus' and ' $\mathrm{A} / \mathrm{B}$ glomerulus' during $\mathrm{A}+$ learning). $\mathrm{A}$ similar mechanism mediates associative LTP in sensory neurons in Aplysia with serotonin as reinforcer for synaptic plasticity (Carew et al., 1984). (ii) Reinforcerindependent Hebbian LTP (coincident pre- and postsynaptic activity) and long-term depression (LTD; presynaptic activity that does not coincide with postsynaptic activity; Stent, 1973) at inhibitory LN-toORN synapses. Such bidirectional plasticity at inhibitory synapses has been found in the cerebellum (Aizenman et al., 1998) - coincident preand postsynaptic activity leads to LTP, presynaptic and no postsynaptic activity leads to LTD. We propose the same bidirectional plasticity for LN-to-ORN synapses. This mechanism would modify the AL network based on odour-driven activity statistics, and would therefore function as an adaptive gain-control filter. ORN-to-PN transmission is amplified in glomeruli with weak input whereas it is weakened in glomeruli with strong input. After learning, the two postulated synaptic changes would sum up and result in an increased ORN-to-PN transmission in 'A glomeruli' and 'none glomeruli', no change in ' $\mathrm{B}$ glomeruli', and decreased transmission in 'A/B glomeruli' (Fig. 4C).

What might be the adaptive value of glomerulus-specific response changes? The increase in 'A glomeruli' might facilitate the detection of the learned odour $A$ and chemically similar odours that activate the same ORNs. The 'A/B glomeruli' carry ambiguous information as they respond to both the rewarded $\mathrm{A}$ and unrewarded $\mathrm{B}$. The decrease in responses after training may thus reduce ambiguity and may facilitate discrimination between A and B. In line with this hypothesis, we found a training-induced increase in the distance between the $\mathrm{A}$ and $\mathrm{B}$ patterns in discriminators but not in non-discriminators (Fig. 2B). The elevation of odour responses in 'none glomeruli' might reflect an unspecific increased sensitivity to odours in general. Taken together our results support the idea that learning modifies the functional AL circuitry, which ameliorates odour representations and thereby improves the bees' ability to detect and discriminate learned odours. Given the high functional similarity in olfactory networks across species it is tempting to speculate that similar mechanisms also occur in the mammalian olfactory bulb.

\section{Acknowledgements}

We thank Ana Florencia Silbering for providing analysis routines for the behavioural data and for statistical advice; Stephanie Biergans and Nadine Treiber for help with the behavioural experiments; Thomas Nowotny, the entire Galizia group for fruitful discussions; and two anonymous reviewers for valuable suggestions. This work was supported by Bundesministerium für Bildung und Forschung (BMBF; 01GQ0931 to P.S. and C.G.G.) and Deutsche Forschungsgemeinschaft (DFG; SPP1392 to C.G.G.)

\section{Abbreviations}

$\mathrm{A}+$, odour $\mathrm{A}$ paired with the unconditioned stimulus; $\mathrm{AL}$, antennal lobe; $\mathrm{B}-$, unpaired odour B; 1-APT, lateral antenna-protocerebral tract; LN, local interneuron; LTD, long-term depression; LTP, long-term potentiation; m-APT, medial antenna-protocerebral tract; ORN, olfactory receptor neuron; PER, proboscis extension reflex; PN, projection neuron.

\section{References}

Abel, R., Rybak, J. \& Menzel, R. (2001) Structure and response patterns of olfactory interneurons in the honcy bee, Apis mellifera. J. Comp. Neurol., 437, 363-383.

Aizenman, C.D., Manis, P.B. \& Linden, D.J. (1998) Polarity of long-term synaptic gain change is related to postsynaptic spike firing at a cercbellar inhibitory synapse. Neuron, 21, 827-835. 
Arenas, A., Giurfa, M., Farina, W.M. \& Sandoz, J. (2009) Early olfactory experience modifies neural activity in the antennal lobe of a social insect at the adult stage. Eur: J. Neurosci., 30, 1498-1508.

Blum, A., Li, W., Cressy, M. \& Dubnau, J. (2009) Short- and long-term memory in Drosophila require cAMP signaling in distinct neuron types. Curr Biol., 19, 1341-1350.

Carew, T.J., Hawkins, R.D., Abrams, T.W. \& Kandel, E.R. (1984) A test of Hebb's postulate at identified synapses which mediate classical conditioning in Aplysia. J. Neurosci., 4, 1217-1224.

Daly, K.C., Christensen, T.A., Smith, B.H., Lei, H. \& Hildebrand, J.G. (2004) Learning modulates the ensemble representations for odors in primary olfactory networks. Proc. Natl Acad. Sci. USA, 101, 10476-10481.

Davis, R.L. (2004) Olfactory learning. Neuron, 44, 31-48.

Deisig, N., Giurfa, M. \& Sandoz, J. (2010) Antennal lobe processing increases separability of odor mixture representations in the honey bee. $J$. Neurophys iol., 103, 2185-2194.

Denker, M., Finke, R., Schaupp, F., Grün, S. \& Menzel, R. (2010) Neural correlates of odor learning in the honey bee antennal lobe. Eur. J. Neurosci. 31, 119-133.

Doucette, W. \& Restrepo, D. (2008) Profound context-dependent plasticity of mitral cell responses in olfactory bulb. PLoS Biol., 6, e258.

Faber, T. \& Menzel, R. (2001) Visualizing mushroom body response to a conditioned odor in honey bees. Naturwissenschaften, 88, 472-476.

Faber, T., Joerges, J. \& Menzel, R. (1999) Associative learning modifies neural representations of odors in the insect brain. Nat. Neurosci., 2, 74-78.

Fernandez, P.C., Locatelli, F.F., Person-Rennell, N., Deleo, G. \& Smith, B.H (2009) Associative conditioning tunes transient dynamics of early olfactory processing. J. Neurosci., 29, 10191-10202.

Fiala, A. (2007) Olfaction and olfactory learning in Drosophila: recent progress. Curr: Opin. Neurobiol., 17, 720-726.

Galizia, G. (2008) Insect olfaction. In Basbaum, A.I., Kancko, A., Shepherd G.M. \& Westheimer, G. (Eds), The Senses: A Comprehensive Reference, Vol 4: Olfaction \& Taste (Firestein, S, Beauchamp, GK, Eds). Academic Press, San Diego, CA, pp. 725-770

Galizia, C.G. \& Rössler, W. (2009) Parallel olfactory systems in insects: anatomy and function. Annu. Rev. Entomol., 55, 399 420.

Galizia, C.G., McIllwrath, S.L. \& Menzel, R. (1999a) A digital threedimensional atlas of the honey bee antennal lobe based on optical sections acquired by confocal microscopy. Cell Tissue Res., 295, 383-394.

Galizia, C.G., Sachse, S., Rappert, A. \& Menzel, R. (1999b) The glomerular code for odor representation is species specific in the honey bee Apis mellifera. Nat. Neurosci., 2, 473-478.

Giurfa, M. (2007) Behavioral and neural analysis of associative learning in the honeybee: a taste from the magic well. J. Comp. Physiol. A Neuroethol. Sens. Neural. Behav. Physiol., 193, 801-824

Grünbaum, L. \& Müller, U. (1998) Induction of a specific olfactory memory leads to a long-lasting activation of protein kinase $\mathrm{C}$ in the antennal lobe of the honey bec. J. Neurosci., 18, 4384-4392.

Hammer, M. (1993) An indentified neuron mediates the unconditioned stimulus in associative olfactory learning in honcy bees. Nature, 366, 59-63.

Hammer, M. \& Menzel, R. (1995) Learning and memory in the honey bee. J. Neurosci., 15, 1617-1630.

Hammer, M. \& Menzel, R. (1998) Multiple sites of associative odor learning as revealed by local brain microinjections of octopamine in honeybees. Learn. Mem., 5, 146-156.

Hebb, D.O. (1949) The Organization of Behavior: A Neuropsychological Theory. Wiley, New York.

Heisenberg, M. (2003) Mushroom body memoir: from maps to models. Nat. Rev. Neurosci., 4, 266-275.

Hildebrand, J.G. \& Shepherd, G.M. (1997) Mechanisms of olfactory discrimination: converging cvidence for common principles across phyla. Annu. Rev. Neurosci., 20, 595-631.

Kay, L.M. \& Laurent, G. (1999) Odor- and context-dependent modulation of mitral cell activity in behaving rats. Nat. Neurosci., 2, 1003-1009.

Kirschner, S., Kleineidam, C.J., Zube, C., Rybak, J., Grünewald, B. \& Rössler, W. (2006) Dual olfactory pathway in the honey bee, Apis mellifera. J. Comp. Neurol., 499, 933-952.

Kreissl, S., Strasser, C. \& Galizia, C.G. (2010) Allatostatin immunoreactivity in the honey bee brain. J. Comp. Neurol., 518, 1391-1417.

Linster, C., Sachse, S. \& Galizia, C.G. (2005) Computational modeling suggests that response properties rather than spatial position determine connectivity between olfactory glomeruli. J. Neurophysiol., 93, 3410 3417

Lunney, G.H. (1970) Using analysis of variance with a dichotomous dependent variable: an empirical study. J. Educ. Meas., 7, 263-269.
Menzel, R. (2001) Searching for the memory trace in a mini-brain, the honeybec. Learn. Mem., 8, 53-62.

Menzel, R. \& Bitterman, M.E. (1983) Learning by honeybees in an unnatural situation. In Huber, F. \& Markl, H. (Eds), Neuroethology and Behavioral Physiology. Springer, New York, pp. 206-215.

Menzel, R., Galizia, G., Müller, D. \& Szyszka, P. (2005) Odor coding in projection neurons of the honeybee brain. Chem. Senses, 30(Suppl 1), i301-i302.

Okada, R., Rybak, J., Manz, G. \& Menzel, R. (2007) Learning-related plasticity in PE1 and other mushroom body-extrinsic neurons in the honeybee brain. J. Neurosci., 27, 11736-11747.

Olsen, S.R. \& Wilson, R.I. (2008) Lateral presynaptic inhibition mediates gain control in an olfactory circuit. Nature, 452, 956-960.

Pamir, E., Gehring, K., Stollhoff, N., Eisenhardt, D. \& Nawrot, M.P. (2010) History matters - a sequential analysis of the PER conditioning in honeybees. Front. Comput. Neurosci., Conference Abstract: Bernstein Conference on Computational Neuroscience. doi: 10.3389/conf.fncom.2010.51.00031

Peele, P., Ditzen, M., Menzel, R. \& Galizia, C.G. (2006) Appetitive odor learning does not change olfactory coding in a subpopulation of honeybec antennal lobe neurons. J. Comp. Physiol. A., 192, 1083-1103.

Perez-Orive, J., Mazor, O., Turner, G.C., Cassenaer, S., Wilson, R.I. \& Laurent, G. (2002) Oscillations and sparsening of odor representations in the mushroom body. Science, 297, 359-365.

Roussel, E., Sandoz, J.C. \& Giurfa, M. (2010) Searching for learning dependent changes in the antennal lobe: simultaneous recording of neural activity and aversive olfactory learning in honey bees. Front. Behav Neurosci., 4, 155.

Sachse, S. \& Galizia, C.G. (2002) Role of inhibition for temporal and spatial odor representation in olfactory output neurons: a calcium imaging study. J. Neurophysiol., 87, 1106-1117.

Sachse, S., Rappert, A. \& Galizia, C.G. (1999) The spatial representation of chemical structures in the antennal lobe of honeybees: steps towards the olfactory code. Eur. J. Neurosci., 11, 3970-3982

Sandoz, J., Galizia, C.G. \& Menzel, R. (2003) Side-specific olfactory conditioning leads to more specific odor representation between sides but not within sides in the honeybec antennal lobes. Neuroscience, 120, 1137-1148.

Schwaerzel, M. \& Müller, U. (2006) Dynamic memory networks: dissecting molecular mechanisms underlying associative memory in the tempora domain. Cell. Mol. Life Sci., 63, 989-998.

Schwacrzel, M., Monastirioti, M., Scholz, H., Friggi-Grelin, F., Birman, S. \& Heisenberg, M. (2003) Dopamine and octopamine differentiate between aversive and appetitive olfactory memories in Drosophila. J. Neurosci., 23, 10495-10502.

Shang, Y., Claridge-Chang, A., Sjulson, L., Pypaert, M. \& Miesenböck, G. (2007) Excitatory local circuits and their implications for olfactory processing in the fly antennal lobe. Cell, 128, 601-612.

Silbering, A.F. \& Galizia, C.G. (2007) Processing of odor mixtures in the Drosophila antennal lobe reveals both global inhibition and glomerulusspecific interactions. J. Neurosci., 27, 11966-11977.

Silbering, A.F., Okada, R., Ito, K. \& Galizia, C.G. (2008) Olfactory information processing in the Drosophila antennal lobe: anything gocs? J. Neurosci., 28 13075-13087.

Stent, G.S. (1973) A physiological mechanism for Hebb's postulate of learning Proc. Natl. Acad. Sci. U.S.A., 70, 997-1001.

Stollhoff, N., Menzel, R. \& Eisenhardt, D. (2005) Spontaneous recovery from extinction depends on the reconsolidation of the acquisition memory in an appetitive learning paradigm in the honeybee (Apis mellifera). $J$. Neurosci., 25, 4485-4492.

Stollhoff, N., Menzel, R. \& Eisenhardt, D. (2008) One retrieval trial induces reconsolidation in an appetitive learning paradigm in honeybees (Apis mellifera). Neurobiol. Learn. Mem., 89, 419-425.

Szyszka, P., Galkin, A. \& Menzel, R. (2008) Associative and non-associative plasticity in kenyon cells of the honey bec mushroom body. Fron. Syst Neurosci., 2, 3 .

Szyszka, P., Demmler, C., Oemisch, M., Sommer, L., Biergans, S., Birnbach, B., Silbering, A.F. \& Galizia, C.G. (2011) Mind the gap: olfactory trace conditioning in honeybees. J. Neurosci., 31, 7229-7239.

Thompson, R.F. (2005) In search of memory traces. Annu. Rev. Psychol., 56, 1-23.

Thum, A.S., Jenett, A., Ito, K., Heisenberg and M., Tanimoto, H. (2007) Multiple memory traces for olfactory reward learning in Drosophila J. Neurosci, 27, 11132-11138.

Wilson, R.I., Turner, G.C. \& Laurent, G. (2004) Transformation of olfactory representations in the Drosophila antennal lobe. Science, 303, 366-370.

Yu, D., Ponomarev, A. \& Davis, R.L. (2004) Altered representation of the spatial code for odors after olfactory classical conditioning: memory trace formation by synaptic recruitment. Neuron, 42, 437-449. 